PROCEEDINGS OF THE

AMERICAN MATHEMATICAL SOCIETY

Volume 140, Number 3, March 2012, Pages 977-986

S 0002-9939(2011)10964-X

Article electronically published on August 31, 2011

\title{
WIENER TYPE THEOREMS FOR JACOBI SERIES WITH NONNEGATIVE COEFFICIENTS
}

\author{
H. N. MHASKAR AND S. TIKHONOV \\ (Communicated by Walter Van Assche)
}

\begin{abstract}
This paper gives three theorems regarding functions integrable on $[-1,1]$ with respect to Jacobi weights and having nonnegative coefficients in their (Fourier-) Jacobi expansions. We show that the $L^{p}$-integrability (with respect to the Jacobi weight) on an interval near 1 implies the $L^{p}$-integrability on the whole interval if $p$ is an even integer. The Jacobi expansion of a function locally in $L^{\infty}$ near 1 is shown to converge uniformly and absolutely on $[-1,1]$; in particular, such a function is shown to be continuous on $[-1,1]$. Similar results are obtained for functions in local Besov approximation spaces.
\end{abstract}

\section{INTRODUCTION}

A well-known theorem by Norbert Wiener states (see e.g. [5. pp. 242, 250]) that if $f$ is a $2 \pi$-periodic function in $L^{1}(-\pi, \pi)$ with nonnegative Fourier coefficients $c_{n}(f) \geq 0$ and $f \in L^{2}(-\delta, \delta)$ for some $\delta>0$, then $f \in L^{2}(-\pi, \pi)$. Since the Fourier coefficients of $|f|^{2}$ are given formally by $\sum_{k \in \mathbb{Z}} c_{n-k}(f) \overline{c_{k}(f)}$, it is easy to deduce that a similar conclusion holds also when the $L^{2}$ norms are replaced by $L^{p}$ norms, $p=2,4,6, \cdots$. For $1 \leq p \leq \infty$, let

$$
\begin{gathered}
L_{\mathrm{loc},+}^{p}:=\left\{f \in L^{1}(-\pi, \pi): f \sim \sum_{n \in \mathbb{Z}} c_{n}(f) e^{i n \circ}, c_{n}(f) \geq 0 \text { for every } n \in \mathbb{Z},\right. \\
\text { and there exists } \left.\delta>0 \text { such that } f \in L^{p}(-\delta, \delta)\right\} .
\end{gathered}
$$

Wiener's theorem can now be formulated more concisely as follows: For even positive integer values of $p, L_{\mathrm{loc},+}^{p} \subset L^{p}(-\pi, \pi)$. Wainger [16] and Shapiro [12] have given counterexamples to show that such an inclusion is not true if $p$ is not an even positive integer.

Even though the inclusion $L_{\text {loc, }+}^{p} \subset L^{p}(-\pi, \pi)$ does not hold in general, the variation stated in the following Theorem 1.1 was proved in [1. A subspace $X \subset$ $L^{1}(-\pi, \pi)$ is called solid if $f, g \in L^{1}(-\pi, \pi),\left|c_{n}(f)\right| \leq c_{n}(g), n \in \mathbb{Z}$, and $g \in X$ imply that $f \in X$. (Some authors use the phrase "with upper majorant property"

Received by the editors February 27, 2010 and, in revised form, December 19, 2010.

2010 Mathematics Subject Classification. Primary 33C45, 42C10; Secondary 46E30.

Key words and phrases. Fourier-Jacobi expansion, non-negative coefficients, Besov spaces.

The research of the first author was supported in part by grant DMS-0908037 from the National Science Foundation and grant W911NF-09-1-0465 from the U.S. Army Research Office.

The research of the second author was supported in part by grants MTM2008-05561-C0202/MTM, 2009 SGR 1303, RFFI 09-01-00175, and NSH3252.2010.1. 
instead of solid.) For example, if $1<p \leq 2$ and $p^{\prime}$ is its conjugate exponent, then the Hausdorff-Young inequality [17, Chapter XII, (2.3)] implies that

$$
L^{p}(-\pi, \pi) \subset\left\{f \in L^{1}(-\pi, \pi): \sum_{n \in \mathbb{Z}}\left|c_{n}(f)\right|^{p^{\prime}}<\infty\right\}=: \ell^{p^{\prime}} .
$$

Similarly, a result of Hardy and Littlewood (cf. [17, Chapter XII, (3.19)]) shows that if $1<p \leq 2$, then

$$
L^{p}(-\pi, \pi) \subset\left\{f \in L^{1}(-\pi, \pi): \sum_{n \in \mathbb{Z}}(|n|+1)^{p-2}\left|c_{n}(f)\right|^{p}<\infty\right\}=: H L^{p} .
$$

Clearly, both $\ell^{p^{\prime}}$ and $H L^{p}$ are solid spaces. Other similar examples can be found in [1].

Theorem 1.1. Let $X$ be a solid space, $L^{p}(-\pi, \pi) \subseteq X$. Then

$$
L_{\mathrm{loc},+}^{p} \subseteq X
$$

In the case when $p$ is an even positive integer, the space $L^{p}(-\pi, \pi)$ is itself a solid space, and hence Theorem 1.1 is a generalization of Wiener's theorem.

In the case when $p=\infty$, Paley [11] observed that if $f \in L_{\mathrm{loc},+}^{\infty}$ and $f$ is an even function, then $f$ is continuous on $[-\pi, \pi]$ and its Fourier series converges uniformly and absolutely. In fact, certain smoothness properties of such functions can be characterized in terms of their Fourier coefficients. For example, a theorem of Lorentz [9, Section 4] implies immediately the following result: Let $f(x) \sim$ $\sum_{n \geq 0} c_{n} \cos n x, c_{n} \geq 0$, and assume further that $\left(c_{n}\right)_{n \geq 0}$ is a monotone sequence. Let $E_{n}(f)$ be the best approximation of $f$ (in the supremum norm) by trigonometric polynomials of order $n$, and let $\gamma \in(0,1)$. Then $\limsup _{n \rightarrow \infty} n^{\gamma} E_{n}(f)<\infty$ if and only if $\limsup _{n \rightarrow \infty} n^{\gamma+1} c_{n}<\infty$. Extensions of this result to the case of Besov and Besov-Nikolskii spaces have been given in [7, 2, 14, so as to include the case of $L^{p}$, $1<p<\infty$, as well.

Our main goal in this paper is to present analogues of the above results for functions on $[-1,1]$ and their Jacobi polynomial expansions. We must defer the precise statements to Section 3 after the necessary notation and definitions are given, but we will describe here briefly the results and ideas to the extent we can. We will consider a class $\mathbb{P}$ of functions with nonnegative Jacobi coefficients, where the parameters of the Jacobi polynomials are assumed to be $\geq-1 / 2$. The role of 0 in the definition of local spaces in the context of trigonometric series will now be played by 1. In Theorem 3.1, we show that if $X$ is a solid space (defined based on Jacobi coefficients) and if $f \in \mathbb{P}$ locally in $X$ in a neighborhood of 1 , then $f \in X$. In Theorem 3.4 we will prove that the Jacobi expansion of a function in $\mathbb{P}$ that is locally essentially bounded near 1 is absolutely and uniformly convergent on $[-1,1]$, so that the function is not just essentially bounded but continuous on $[-1,1]$. In Theorem 3.6, we state a refinement of this result where Besov spaces and their local analogues are considered rather than mere continuity. Our results make extensive use of the product and linearization formula for Jacobi polynomials $([3,8)$ as well as the summability and frame operators for Jacobi expansions ([10]).

In Section 2 we review some necessary facts regarding Jacobi polynomials and introduce some notation. The main theorems are stated and proved in Section 3 ,

In order to avoid unnecessarily complicated notation, the symbols used in this introduction will have different meanings in the rest of this paper. 


\section{JACOBI POLYNOMIALS}

We will denote the set of all nonnegative integers by $\mathbb{N}_{0}=\{0,1,2, \cdots\}$. If $n \in \mathbb{N}_{0}$, we will denote by $\Pi_{n}$ (respectively, $\Pi_{n / 2}$ ) the class of all polynomials of degree at most $n$ (respectively, $n / 2$ ). Let $\alpha, \beta>-1$, and let

$$
w_{\alpha, \beta}(x):= \begin{cases}(1-x)^{\alpha}(1+x)^{\beta}, & \text { if }-1<x<1 \\ 0, & \text { otherwise }\end{cases}
$$

For $1 \leq p<\infty$ the space $L^{p}=L^{p}(\alpha, \beta)$ is defined as the space of (equivalence classes of) functions $f$ with

$$
\|f\|_{\alpha, \beta ; p}:=\left(\int_{-1}^{1}|f(x)|^{p} w_{\alpha, \beta}(x) d x\right)^{1 / p}<\infty .
$$

The space of all continuous real-valued functions on $[-1,1]$, equipped with the supremum norm, will be denoted by $C$, and the supremum norm of $f \in C$ will be denoted by $\|f\|_{\infty}$. The space of all infinitely often differentiable $f:[-1,1] \rightarrow \mathbb{C}$ will be denoted by $C^{\infty}$.

There exists a unique system of (Jacobi) polynomials $\left(R_{k}^{(\alpha, \beta)} \in \Pi_{k}\right)_{k \in \mathbb{N}_{0}}$ such that for integer $k, \ell \in \mathbb{N}_{0}, R_{k}^{(\alpha, \beta)}(1)=1$ and

$$
\int_{-1}^{1} R_{k}^{(\alpha, \beta)}(x) R_{\ell}^{(\alpha, \beta)}(x) w_{\alpha, \beta}(x) d x= \begin{cases}\rho_{k}, & \text { if } k=\ell, \\ 0, & \text { otherwise }\end{cases}
$$

where

$$
\rho_{k}=\left\|R_{k}\right\|_{\alpha, \beta ; 2}^{2}=\frac{2^{\alpha+\beta+1} \Gamma(\alpha+1)^{2}}{2 k+\alpha+\beta+1} \frac{\Gamma(k+1) \Gamma(k+\beta+1)}{\Gamma(k+\alpha+1) \Gamma(k+\alpha+\beta+1)} .
$$

The uniqueness of the system implies that $R_{k}{ }^{(\beta, \alpha)}(x)=R_{k}{ }^{(\alpha, \beta)}(-x) / R_{k}{ }^{(\alpha, \beta)}(-1)$, $x \in \mathbb{R}, k \in \mathbb{N}_{0}$. Therefore, we may assume in the sequel that $\alpha \geq \beta$. We will assume also that $\alpha \geq \beta \geq-1 / 2$. Note that the latter assumption will be used further (see formula $(2.5)$ ).

For $f \in L^{1}$, we may define the Jacobi coefficients by

$$
\hat{f}(k):=\hat{f}(\alpha, \beta ; k):=\rho_{k}^{-1} \int_{-1}^{1} f(y) R_{k}^{(\alpha, \beta)}(y) w_{\alpha, \beta}(y) d y, \quad k \in \mathbb{N}_{0} .
$$

Then the formal Jacobi expansion of $f$ has the form $\sum_{k=0}^{\infty} \hat{f}(k) R_{k}(x)$.

Next, we need formulas for functions $f * g$ such that $\hat{f * g}(k)=\hat{f}(k) \hat{g}(k), k \in \mathbb{N}_{0}$, and for the Jacobi coefficients of the product $f g$. Following Koornwinder [8, if $x, y \in[-1,1], r \in[0,1]$, and $\psi \in[0, \pi]$, let

$Z(x, y ; r, \psi)=\frac{1}{2}(1+x)(1+y)+\sqrt{1-x^{2}} \sqrt{1-y^{2}} r \cos \psi+\frac{1}{2}(1-x)(1-y) r^{2}-1$.

We observe that

$$
\begin{aligned}
Z(x, y ; r, \psi)=\frac{1}{2}( & \sqrt{(1+x)(1+y)}-r \sqrt{(1-x)(1-y)})^{2} \\
& +r \sqrt{1-x^{2}} \sqrt{1-y^{2}}(1+\cos \psi)-1 \geq-1 .
\end{aligned}
$$


If $\theta, \varphi \in[0, \pi]$ such that $x=\cos \theta, y=\cos \varphi$, then

$$
\begin{aligned}
& Z(x, y ; r, \psi)=\frac{1}{2}(1+x)(1+y)+\frac{1}{2}(1-x)(1-y)+\sqrt{1-x^{2}} \sqrt{1-y^{2}} \\
& +\frac{1}{2}(1-x)(1-y)\left(r^{2}-1\right)+\sqrt{1-x^{2}} \sqrt{1-y^{2}}(r \cos \psi-1)-1 \\
& =x y+\sqrt{1-x^{2}} \sqrt{1-y^{2}}+\frac{1}{2}(1-x)(1-y)\left(r^{2}-1\right)+\sqrt{1-x^{2}} \sqrt{1-y^{2}}(r \cos \psi-1),
\end{aligned}
$$

and we have

$$
\begin{aligned}
1-Z(x, y ; r, \psi)=1- & \left(x y+\sqrt{1-x^{2}} \sqrt{1-y^{2}}\right)+\frac{1}{2}(1-x)(1-y)\left(1-r^{2}\right) \\
& +(1-r \cos \psi) \sqrt{1-x^{2}} \sqrt{1-y^{2}} \\
\geq & 1-\left(x y+\sqrt{1-x^{2}} \sqrt{1-y^{2}}\right)=1-\cos (\theta-\varphi) \geq 0 .
\end{aligned}
$$

Thus, $Z(x, y ; r, \psi) \in[-1,1]$ for all $x, y \in[-1,1], r \in[0,1]$ and $\psi \in[0, \pi]$.

For $\alpha \geq \beta \geq-1 / 2$, Koornwinder 8 , has proved that there exists a probability measure $\nu^{(\alpha, \beta)}$ on $[0,1] \times[0, \pi]$ such that we have

$$
R_{n}^{(\alpha, \beta)}(x) R_{n}^{(\alpha, \beta)}(y)=\int_{0}^{\pi} \int_{0}^{1} R_{n}^{(\alpha, \beta)}(Z(x, y ; r, \psi)) d \nu^{(\alpha, \beta)}(r, \psi), \quad n \in \mathbb{N}_{0} .
$$

An interesting consequence of (2.5) is the following. For almost all $x, y \in[-1,1]$ and $f \in L^{1}$, let

$$
\mathcal{T}_{y} f(x)=\int_{0}^{\pi} \int_{0}^{1} f(Z(x, y ; r, \psi)) d \nu^{(\alpha, \beta)}(r, \psi) .
$$

Then it is clear that for $x, y \in[-1,1], \mathcal{T}_{y} f(x)=\mathcal{T}_{x} f(y)$ and

$$
\widehat{\mathcal{T}_{y} f}(k)=\hat{f}(k) R_{k}{ }^{(\alpha, \beta)}(y) .
$$

The corresponding convolution operator is defined by

$$
(f * g)(x):=\int_{-1}^{1} f(y) \mathcal{T}_{y} g(x) w_{\alpha, \beta}(y) d y, \quad f, g \in L^{1} .
$$

We have

$$
\widehat{f * g}(k)=\widehat{f}(k) \widehat{g}(k), \quad k \in \mathbb{N}_{0}, f, g \in L^{1} .
$$

Dual to the product formula (2.5) is a linearization formula (see [3]):

$$
R_{n}^{(\alpha, \beta)}(x) R_{m}^{(\alpha, \beta)}(x)=\sum_{k=|n-m|}^{n+m} g^{(\alpha, \beta)}(n, m ; k) R_{k}^{(\alpha, \beta)}(x)
$$

for all $n, m \in \mathbb{N}_{0}, x \in[-1,1]$. The coefficients $g^{(\alpha, \beta)}(n, m ; k)$ are nonnegative for all $k, n, m \in \mathbb{N}_{0}$ and, moreover,

$$
\sum_{k=|n-m|}^{n+m} g^{(\alpha, \beta)}(n, m ; k)=1
$$

Thus, we may think of $\left(g^{(\alpha, \beta)}(n, m ; k)\right)_{k}$ as a probability distribution on a subset of $\mathbb{N}_{0}$. We note also that

$$
g^{(\alpha, \beta)}(n, m ; k)=\rho_{k}^{-1} \int_{-1}^{1} R_{n}^{(\alpha, \beta)}(y) R_{m}^{(\alpha, \beta)}(y) R_{k}^{(\alpha, \beta)}(y) w_{\alpha, \beta}(y) d y .
$$


In particular,

$$
g^{(\alpha, \beta)}(k, 0 ; k)=\rho_{k}^{-1} \int_{-1}^{1}\left\{R_{k}^{(\alpha, \beta)}(y)\right\}^{2} w_{\alpha, \beta}(y) d y=1 .
$$

Just as (2.5) can be used via (2.6) to define a generalized convolution of two functions, (2.9) can be used to define a convolution of sequences. If $\mathbf{a}=\left(a_{k}\right)_{k \in \mathbb{N}_{0}}$ and $\mathbf{b}=\left(b_{k}\right)_{k \in \mathbb{N}_{0}}$, we define formally

$$
(\mathbf{a} * \mathbf{b})(k)=\sum_{n, m=0}^{\infty} g^{(\alpha, \beta)}(n, m ; k) a_{n} b_{m} .
$$

Analogous to (2.8) and the classical Cauchy formula for the products of power series, we have the following formula for products of formal Jacobi expansions:

$$
\left(\sum_{n=0}^{\infty} a_{n} R_{n}{ }^{(\alpha, \beta)}(x)\right)\left(\sum_{m=0}^{\infty} b_{m} R_{m}{ }^{(\alpha, \beta)}(x)\right)=\sum_{k=0}^{\infty}(\mathbf{a} * \mathbf{b})(k) R_{k}{ }^{(\alpha, \beta)}(x), \quad x \in[-1,1] .
$$

\section{MAIN RESUlts}

In the sequel, we consider $\alpha \geq \beta \geq-1 / 2$ to be fixed parameters. Let $\mathbb{P}$ be the class of all $f \in L^{1}$ such that $\hat{f}(k) \geq 0$ for $k \in \mathbb{N}_{0}$. It is well known that in the theory of Jacobi expansions, the point 1 plays the role of 0 in the theory of Fourier series. Thus, if $T$ is a trigonometric polynomial with nonnegative Fourier coefficients, then $\max _{x \in[-\pi, \pi]}|T(x)|=T(0)$. Similarly, in view of [13, Theorem 7.32.1],

$$
\max _{x \in[-1,1]}\left|R_{k}^{(\alpha, \beta)}(x)\right|=R_{k}^{(\alpha, \beta)}(1)=1 .
$$

Therefore, it is easy to verify that for every $P \in \mathbb{P} \cap\left(\bigcup_{n=0}^{\infty} \Pi_{n}\right)$ and $a \in[-1,1)$,

$$
\max _{x \in[-1,1]}|P(x)|=P(1)=\max _{x \in[a, 1]}|P(x)| .
$$

Accordingly, we define the analogues of local spaces as follows. If $Y$ is any subspace of $L^{1}$, then $Y_{\text {loc }}$ is the class of all $f \in L^{1}$ with the following property: there exists a nondegenerate interval $I \subseteq[-1,1]$ with $1 \in I$ such that for any $\phi \in C^{\infty}$ supported on $I, f \phi \in Y$. If $f, g \in L^{1}$, we will write $f \preceq g$ if $|\hat{f}(k)| \leq \hat{g}(k)$ for all $k \in \mathbb{N}_{0}$. A subspace $X \subset L^{1}$ is called solid if $f, g \in L^{1}, f \preceq g$, and $g \in X$ imply that $f \in X$. For example, using (2.13), (2.12), and the fact that $g^{(\alpha, \beta)}(n, m ; k) \geq 0$ for all $n, m, k \in \mathbb{N}_{0}$, it is easy to conclude that when $f \preceq g$, then $|f|^{2} \preceq|g|^{2}$. Hence, if $p$ is an even positive integer, then $L^{p}$ is a solid space. Thus, Theorem 3.1 below is the analogue of Wiener's theorem for Jacobi expansions.

Our first main result is the analogue of Theorem 1.1.

Theorem 3.1. Let $X \subset L^{1}$ be a solid space. Then $X_{\text {loc }} \cap \mathbb{P}=X \cap \mathbb{P}$. In particular, if $1<p \leq \infty$ and $L^{p} \subseteq X$, then $L_{\mathrm{loc}}^{p} \cap \mathbb{P} \subseteq X$.

In the case when $p$ is not a positive even integer, we note that using the counterexamples of Wainger and Shapiro, we can always construct a cosine trigonometric series with positive coefficients such that its sum is not $p$-th power integrable on $[0, \pi]$. Since $R_{k}{ }^{(-1 / 2,-1 / 2)}$ are Chebyshev polynomials of the first kind, these counterexamples also demonstrate that with $\alpha=\beta=-1 / 2, L_{\text {loc }}^{p} \cap \mathbb{P} \not \subset L^{p}$ when $p$ is not a positive even integer. 
The proof of Theorem 3.1 relies upon the following two lemmas. If $f \in C$, we define

$$
E_{n}(f):=\min _{P \in \Pi_{n}}\|f-P\|_{\infty} .
$$

Lemma 3.2. Let $f \in C^{\infty}$ and $x \in[-1,1]$. Then the function $x \mapsto \mathcal{T}_{x} f$ is in $C^{\infty}$.

Proof. The well-known direct theorem of approximation theory [15, Section 5.1.5, (22)] implies that for any integer $S \geq 1, E_{n}(f) \leq c_{1}(f, S) n^{-S}$ for some positive constant $c_{1}(f, S)$ independent of $n$. Let $\ell \geq 0$ be an integer. We choose $S>$ $2 \ell+2 \alpha+3$. In view of (2.1) and (3.1), we obtain for $k=2,3, \cdots$ and any $P \in \Pi_{k-1}$,

$$
\begin{aligned}
|\hat{f}(k)|=\rho_{k}^{-1}\left|\int_{-1}^{1} f(t) R_{k}(t) w_{\alpha, \beta}(t) d t\right| & =\rho_{k}^{-1}\left|\int_{-1}^{1}(f(t)-P(t)) R_{k}(t) w_{\alpha, \beta}(t) d t\right| \\
& \leq \rho_{k}^{-1} \rho_{0}\|f-P\|_{\infty} .
\end{aligned}
$$

Thus, (2.2) leads to the estimate $|\hat{f}(k)| \leq c_{2}(S, \alpha, \beta, f) k^{2 \alpha+1-S}$. Moreover, Markov's inequality [15, Section 4.8.62, (32)] implies that $\left\|R_{k}^{(\ell)}\right\|_{\infty} \leq k^{2 \ell}$. Since $S>2 \ell+2 \alpha+3$, it follows that

$$
\sum_{k=2}^{\infty}|\hat{f}(k)|\left\|R_{k}\right\|_{\infty}\left\|R_{k}{ }^{(\ell)}\right\|_{\infty} \leq c_{2}(S, \alpha, \beta, f) \sum_{k=2}^{\infty} k^{2 \alpha+1-S+2 \ell}<\infty .
$$

This completes the proof.

Lemma 3.3. Let $\delta \in(0,1)$. There exists $\phi_{\delta}:[-1,1] \rightarrow[0, \infty)$ such that $\phi_{\delta} \in C^{\infty}$, $\phi_{\delta}(t)=0$ if $-1 \leq t \leq 1-\delta, \phi_{\delta} \in \mathbb{P}$, and $\hat{\phi}_{\delta}(0)=1$. If $f \in \mathbb{P}$, then $f \preceq f \phi_{\delta}$.

Proof. In this proof only, let $\theta_{0} \in(0, \pi / 4)$ be chosen so that $1-\delta=\cos \left(2 \theta_{0}\right)$, and let $g=g_{\delta}:[-1,1] \rightarrow[0, \infty)$ be a function in $C^{\infty}$, supported on $\left[\cos \theta_{0}, 1\right]$, such that $\hat{g}(0)=1$. Let

$$
\begin{aligned}
\phi_{\delta}(x):=(g * g)(x) & =\int_{-1}^{1} g(y) \mathcal{T}_{y} g(x) w_{\alpha, \beta}(y) d y \\
& =\int_{-1}^{1} g(y) \mathcal{T}_{x} g(y) w_{\alpha, \beta}(y) d y, \quad x \in[-1,1] .
\end{aligned}
$$

In view of Lemma $3.2, \phi_{\delta}:[-1,1] \rightarrow[0, \infty)$ is in $C^{\infty}$. Also, in view of (2.8), $\hat{\phi}_{\delta}(k)=(\hat{g}(k))^{2} \geq 0, k \in \mathbb{N}_{0}$, and in particular, $\hat{\phi}_{\delta}(0)=(\hat{g}(0))^{2}=1$.

If $|\theta-\varphi| \geq \theta_{0}$, then (2.4) implies that $Z(x, y ; r, \psi) \leq \cos (\theta-\varphi) \leq \cos \theta_{0}$. Therefore, $g(Z(x, y ; r, \psi))=0$ and hence, $\mathcal{T}_{y} g(x)=0$. Hence, if $J$ denotes the set $\left\{\cos \varphi:|\theta-\varphi| \leq \theta_{0}\right\}$, then

$$
\phi_{\delta}(x)=\int_{J} g(y) \mathcal{T}_{y} g(x) w_{\alpha, \beta}(y) d y .
$$

If $\theta \geq 2 \theta_{0}$, then for $y \in J, 0<\theta_{0} \leq \theta-\theta_{0} \leq \varphi$; i.e., $y=\cos \varphi \leq \cos \theta_{0}$, and $g(y)=0$. Thus, $\phi_{\delta}$ is supported on $\left[\cos \left(2 \theta_{0}\right), 1\right]=[1-\delta, 1]$.

Next, let $f \in \mathbb{P}$. Since $\hat{f}(n) \geq 0$ for all $n \in \mathbb{N}_{0}$, we obtain in view of (2.13), (2.12), 2.11) that for each $k \in \mathbb{N}_{0}$,

$$
\begin{aligned}
\widehat{f \phi_{\delta}}(k) & =\sum_{n, m=0}^{\infty} g^{(\alpha, \beta)}(n, m ; k) \hat{f}(n) \hat{\phi}_{\delta}(m) \\
& \geq g^{(\alpha, \beta)}(k, 0 ; k) \hat{f}(k) \hat{\phi}_{\delta}(0)=\hat{f}(k) .
\end{aligned}
$$

Thus, $f \preceq f \phi_{\delta}$. 
Proof of Theorem 3.1. Let $f \in X_{\mathrm{loc}} \cap \mathbb{P}$ and $\delta \in(0,1)$ be such that $f \phi_{\delta} \in X$, where $\phi_{\delta}$ is as in Lemma 3.3. Since $X$ is solid, $f \preceq f \phi_{\delta}$, and $f \phi_{\delta} \in X$, it follows that $f \in X$ as well.

Next, we consider the case $p=\infty$. First, we state an analogue of the result of Paley.

Theorem 3.4. If $f \in L_{\text {loc }}^{\infty} \cap \mathbb{P}$, then $\sum_{k=0}^{\infty} \hat{f}(k)\left|R_{k}(x)\right|<\infty, x \in[-1,1]$, with the series converging uniformly on $[-1,1]$. In particular, $L_{\mathrm{loc}}^{\infty} \cap \mathbb{P}=C \cap \mathbb{P}$.

The proof of this theorem depends upon the following lemma, which is formulated by many authors; a proof can be found, for example, in [10, Lemma 4.2]. First, we define the de la Vallée-Poussin type operators. Let $h:[0, \infty) \rightarrow[0,1]$ be a $C^{\infty}$, nonincreasing function such that $h(x)=1$ for $[0,1 / 2]$ and $h(x)=0$ for $[1, \infty)$. For $f \in L^{1}, n \geq 0, x \in[-1,1]$, let

$$
\sigma_{n}(h, f, x)=\sum_{k=0}^{n-1} h\left(\frac{k}{n}\right) \hat{f}(k) R_{k}(x)
$$

and

$$
\tau_{n}(h, f)=\sigma_{2 n}(h, f)-\sigma_{n}(h, f) .
$$

Lemma 3.5. Let $f \in C$. Then

$$
\left\|\sigma_{n}(h, f)\right\|_{\infty} \leq c\|f\|_{\infty},
$$

where $c$ is a positive constant depending only on $\alpha, \beta$, and $h$. Moreover, $\sigma_{n}(h, P)=$ $P$ if $P \in \Pi_{n / 2}$, and hence

$$
E_{n}(f) \leq\left\|f-\sigma_{n}(h, f)\right\|_{\infty} \leq(1+c) E_{n / 2}(f) .
$$

Proof of Theorem 3.4. Let $f \in L_{\mathrm{loc}}^{\infty} \cap \mathbb{P}$ and $\delta \in(0,1)$ be such that $f \phi_{\delta} \in L^{\infty}$, where $\phi_{\delta}$ is as in Lemma 3.3. Let $n \geq 1$ be an integer. Since $h$ is a nonnegative function, $f \in \mathbb{P}$, and $f \preceq f \phi_{\delta}$, using (3.1) we see that

$$
h\left(\frac{k}{2 n}\right) \hat{f}(k)\left\|R_{k}\right\|_{\infty}=h\left(\frac{k}{2 n}\right) \hat{f}(k) \leq h\left(\frac{k}{2 n}\right) \widehat{f \phi_{\delta}}(k), \quad k \in \mathbb{N}_{0} .
$$

Since $h(t)=1$ for $0 \leq t \leq 1 / 2$, we obtain using (3.2) and (3.7) that for every integer $n \geq 1$,

$$
\begin{aligned}
\sum_{k=0}^{n} \hat{f}(k)\left\|R_{k}\right\|_{\infty} & \leq \sum_{k=0}^{\infty} h\left(\frac{k}{2 n}\right) \hat{f}(k)\left\|R_{k}\right\|_{\infty} \leq \sum_{k=0}^{\infty} h\left(\frac{k}{2 n}\right) \widehat{f \phi_{\delta}}(k) \\
& =\left\|\sigma_{2 n}\left(h, f \phi_{\delta}\right)\right\|_{\infty} \leq c\left\|f \phi_{\delta}\right\|_{\infty}<\infty
\end{aligned}
$$

This completes the proof.

Finally, we discuss a variant for Besov spaces, which we now define. Let $0<\rho \leq$ $\infty, \gamma>0$, and $\mathbf{a}=\left(a_{n}\right)_{n \in \mathbb{N}_{0}}$ be a sequence of real numbers. We define a sequence of space as

$$
\|\mathbf{a}\|_{\rho, \gamma}:=\left\{\begin{array}{lll}
{\left[\sum_{n=0}^{\infty} 2^{n \gamma \rho}\left|a_{n}\right|^{\rho}\right]^{1 / \rho},} & \text { if } & 0<\rho<\infty \\
\sup _{n \geq 0} 2^{n \gamma}\left|a_{n}\right|, & \text { if } & \rho=\infty
\end{array}\right.
$$


The space of sequences a for which $\|\mathbf{a}\|_{\rho, \gamma}<\infty$ will be denoted by $\mathrm{b}_{\rho, \gamma}$. For $0<\rho \leq \infty, \gamma>0$, the Besov space $B_{\rho, \gamma}$ consists of functions $f \in L^{\infty}$ for which the sequence $\left(E_{2^{n}}(f)\right)_{n \in \mathbb{N}_{0}} \in \mathrm{b}_{\rho, \gamma}$. We have proved in [10, Theorem 2.1] that $f \in B_{\rho, \gamma}$ if and only if $\left(\left\|\tau_{2^{n}}(h, f)\right\|_{\infty}\right)_{n \in \mathbb{N}_{0}} \in \mathrm{b}_{\rho, \gamma}$.

The following theorem is a refinement of Theorem 3.4. Let $S_{n}(f)$ be the $n$-th partial sum of the Jacobi expansion of $f$, i.e., $S_{n}(f)=\sum_{k=0}^{n-1} \hat{f}(k) R_{k}$.

Theorem 3.6. Let $0<\rho \leq \infty, \gamma>0$. For $f \in \mathbb{P}$ the following conditions are equivalent:

(a) $\quad f \in\left(B_{\rho, \gamma}\right)_{\text {loc }}$,

(b) $\quad f \in B_{\rho, \gamma}$,

(c) $\quad\left(\left\|S_{2^{n+1}}-S_{2^{n}}(f)\right\|_{\infty}=\sum_{k=2^{n}}^{2^{n+1}-1} \hat{f}(k)\right)_{n \in \mathbb{N}_{0}} \in \mathrm{b}_{\rho, \gamma}$.

Proof. To prove (a) $\Rightarrow(\mathrm{b})$, we need to show that $\left(B_{\rho, \gamma}\right)_{\text {loc }} \cap \mathbb{P} \subset B_{\rho, \gamma}$. Let $f \in\left(B_{\rho, \gamma}\right)_{\text {loc }} \cap \mathbb{P}$ and $\delta \in(0,1)$ be such that $f \phi_{\delta} \in B_{\rho, \gamma} \cap \mathbb{P}$, where $\phi_{\delta}$ is as in Lemma 3.3. Then $\left(\left\|\tau_{2^{n}}\left(h, f \phi_{\delta}\right)\right\|_{\infty}\right)_{n \in \mathbb{N}_{0}} \in \mathrm{b}_{\rho, \gamma}$. Since $h$ is nonincreasing, we have

$$
g_{k, n}:=h\left(k / 2^{n+1}\right)-h\left(k / 2^{n}\right) \geq 0, \quad k, n \in \mathbb{N}_{0} .
$$

Since $f \preceq f \phi_{\delta}$, this implies that

$$
0 \leq g_{k, n} \hat{f}(k) \leq g_{k, n} \widehat{f \phi_{\delta}}(k), \quad k, n \in \mathbb{N}_{0} .
$$

So, using (3.2), we conclude that for $n \in \mathbb{N}_{0}$,

$$
\begin{aligned}
\left\|\tau_{2^{n}}(h, f)\right\|_{\infty} & =\tau_{2^{n}}(h, f, 1)=\sum_{k=0}^{\infty} g_{k, n} \hat{f}(k) \\
& \leq \sum_{k=0}^{\infty} g_{k, n} \widehat{f \phi_{\delta}}(k)=\left\|\tau_{2^{n}}\left(h, f \phi_{\delta}\right)\right\|_{\infty} .
\end{aligned}
$$

Since $\left(\left\|\tau_{2^{n}}\left(h, f \phi_{\delta}\right)\right\|_{\infty}\right)_{n \in \mathbb{N}_{0}} \in \mathrm{b}_{\rho, \gamma}$, this implies that $\left(\left\|\tau_{2^{n}}(h, f)\right\|_{\infty}\right)_{n \in \mathbb{N}_{0}} \in \mathrm{b}_{\rho, \gamma}$; i.e., $f \in B_{\rho, \gamma}$.

The implication (b) $\Rightarrow$ (a) is clear.

We prove that (a) (alternatively (b)) $\Rightarrow$ (c). Since (a) implies in particular that $f \in L_{\text {loc }}^{\infty} \cap \mathbb{P}$, Theorem 3.4 implies that the series $\sum \hat{f}(k) R_{k}$ converges uniformly and absolutely to $f$. Then, by (3.1) and (3.8), we have

$$
\begin{aligned}
E_{n}(f) & \leq\left\|f-S_{n}(f)\right\|_{\infty} \leq \sum_{k=n}^{\infty} \hat{f}(k) \leq \sum_{k=0}^{\infty}(1-h(k / n)) \hat{f}(k)=f(1)-\sigma_{n}(h, f, 1) \\
& \leq\left\|f-\sigma_{n}(h, f)\right\|_{\infty} \leq(1+c) E_{n / 2}(f) .
\end{aligned}
$$

Thus, $f \in B_{\rho, \gamma}$ if and only if $\left(\left\|f-S_{2^{n}}(f)\right\|_{\infty}\right)_{n \in \mathbb{N}_{0}} \in \mathrm{b}_{\rho, \gamma}$, and secondly, $f \in B_{\rho, \gamma}$ if and only if $\left(\sum_{k=2^{n}}^{\infty} \hat{f}(k)\right)_{n \in \mathbb{N}_{0}} \in \mathrm{b}_{\rho, \gamma}$. In light of the discrete Hardy inequality [6, Lemma 3.4, p. 27], the latter is equivalent to $\left(\sum_{k=2^{n}}^{2^{n+1}-1} \hat{f}(k)\right)_{n \in \mathbb{N}_{0}} \in \mathrm{b}_{\rho, \gamma}$ for any positive $\rho$. 
Remark 1. Assuming additional conditions on the coefficients, we obtain an analogue of Lorentz's result: under the conditions of Theorem 3.6, conditions (a) and (b) are equivalent to the condition $\left(\hat{f}\left(2^{n}\right)\right)_{n \in \mathbb{N}_{0}} \in \mathrm{b}_{\rho, \gamma}$ if $(\hat{f}(n))_{n \in \mathbb{N}_{0}}$ is lacunary or $\left(\hat{f}\left(2^{n}\right)\right)_{n \in \mathbb{N}_{0}} \in \mathrm{b}_{\rho, \gamma+1}$ if $(\hat{f}(n))_{n \in \mathbb{N}_{0}}$ is monotone.

Remark 2. Let $a \geq b \geq-1 / 2$. In [4], Askey and Gasper have given sufficient conditions in order that the connection coefficients $\gamma_{j, k}$ in the expansion below are all nonnegative:

$$
R_{k}^{(\alpha, \beta)}(x)=\sum_{j=0}^{k} \gamma_{j, k} R_{j}^{(a, b)}(x), \quad k \in \mathbb{N}_{0}, x \in \mathbb{R} .
$$

If $f \in C$ and $\hat{f}(\alpha, \beta ; k) \geq 0$ for $k \in \mathbb{N}_{0}$, then (3.8) can be used to deduce easily that

$$
\hat{f}(a, b ; j)=\lim _{n \rightarrow \infty} \sum_{k=j}^{n} \gamma_{j, k} h(k / n) \hat{f}(\alpha, \beta ; k) \geq 0 .
$$

Thus, Theorem 3.4 implies that for $f \in L_{\text {loc }}^{\infty} \cap \mathbb{P}$, the series $\sum|\hat{f}(a, b ; k)|\left\|R_{j}{ }^{(a, b)}\right\|_{\infty}$ converges for all $a \geq b \geq-1 / 2$ for which the connection coefficients in (3.10) are all nonnegative. A similar conclusion can also be made about Theorem 3.6 and Remark 1 above.

\section{REFERENCES}

1. J. M. Ash, S. Tikhonov and J. Tung, Wiener's positive Fourier coefficients theorem in variants of $L^{p}$ spaces, Michigan Math. J. 59, 1 (2010), 143-152. MR2654143

2. R. Askey, Smoothness conditions for Fourier series with monotone coefficients, Acta Sci. Math. (Szeged) 28 (1967), 168-171. MR0212474 (35:3345)

3. R. Askey, Orthogonal Polynomials and Special Functions, Regional Conference Series in Applied Mathematics 21, SIAM, Philadelphia, 1975. MR0481145 (58:1288)

4. R. A. Askey and G. Gasper, Jacobi polynomial expansions of Jacobi polynomials with nonnegative coefficients, Math. Proc. Cambridge Philos. Soc. 70 (1971), 243-255. MR0296369 $(45: 5430)$

5. R. P. Boas, Entire Functions, Academic Press, New York, 1954. MR0068627 (16:914f)

6. R. A. DeVore and G. G. Lorentz, Constructive Approximation, Springer-Verlag, Berlin, 1993. MR:1261635 (95f:41001)

7. A. A. Konyushkov, Best approximations by trigonometric polynomials and Fourier coefficients (Russian), Mat. Sb. N.S. 44(86) (1958), 53-84. MR0096074(20:2571)

8. T. Koornwinder, Jacobi polynomials II. An analytic proof of the product formula, SIAM J. Math. Anal. 5 (1974), 125-137. MR0385198 (52:6063)

9. G. G. Lorentz, Fourier-Koeffizienten und Funktionenklassen, Math. Z. 51 (1948), 135-149. MR0025601 (10:33e)

10. H. N. Mhaskar, Polynomial operators and local smoothness classes on the unit interval, J. Approx. Theory 131 (2004), 243-267. MR2106540 (2005k:41011)

11. R. E. A. C. Paley, On Fourier series with positive coefficients, J. London Math. Soc. 7 (1932), 205-208.

12. H. S. Shapiro, Majorant problems for Fourier coefficients, Quart. J. Math. Oxford (2) 26 (1975), 9-18. MR0372515 (51:8722)

13. G. Szegö, Orthogonal Polynomials, Amer. Math. Soc. Colloq. Publ. 23, Amer. Math. Soc., Providence, 1975. MR 0372517(51:8724)

14. S. Tikhonov, Characteristics of Besov Nikol'skii class of functions, Elec. Trans. Numer. Anal. 19 (2005), 94-104. MR 2149272 (2006d:42002)

15. A. F. Timan, Theory of Approximation of Functions of a Real Variable, English translation, Pergamon Press, 1963. MR0192238 (33:465) 
16. S. Wainger, A problem of Wiener and the failure of a principle for Fourier series with positive coefficients, Proc. Amer. Math. Soc. 20 (1969), 16-18. MR0236397 (38:4693)

17. A. Zygmund, Trigonometric Series, vol. 2, 2nd ed., Cambridge Univ. Press, New York, 1959. MR0107776 (21:6498)

Department of Mathematics, California State University, los Angeles, California 90032

E-mail address: hmhaska@calstatela.edu

ICREA and Centre de Recerca Matemàtica, Campus de Bellaterra, Edifici C, 08193 Bellaterra (BARCelona), Spain

E-mail address: stikhonov@crm.cat 